\title{
Proposal for Magnetic Dichroism With a Standard STEM Probe Beam
}

\author{
Tyler R. Harvey ${ }^{1}$, Vincenzo Grillo ${ }^{2,3}$, Benjamin J. McMorran ${ }^{1}$ \\ 1. Department of Physics, University of Oregon, Eugene, OR USA \\ 2. CNR-Istituto Nanoscienze, Centro S3, Via G. Campi 213/a, Modena, Italy \\ ${ }^{3}$ CNR-IMEM Parco Area delle Scienze 37/A, Parma, Italy
}

No general-purpose technique for atomic-resolution out-of-plane magnetization measurement in the electron microscope yet exists. Electron magnetic circular dichroism (EMCD) comes close, but demands broad illumination and a crystalline specimen [1]. A recent proposal for extension of EMCD might loosen the broad illumination constraint, but still demands crystallinity [2]. Much effort has been dedicated to application of vortex beams towards atomic-resolution magnetic dichroism, but production of monochromatic, coherent, atomic-scale electron vortex beams is challenging [3-6].

We propose a new method to measure magnetic dichroism that requires no special beam preparation nor demands a crystalline specimen. Following our discovery last year of an orbital angular momentumdependent magnetic lensing effect [7], we design a simple dichroism experiment based on measurement of the outgoing orbital angular momentum distribution of an electron beam after interaction with a chiral (e.g. magnetic) specimen.

The key feature of our dichroism experiment - the only moving part — is the polarity of the smallest-bore magnetic lens after the specimen (likely the Lorentz lens for magnetic specimens, or the objective for non-magnetic specimens). A dichroism experiment measures differences in the probabilities of $\Delta \mathrm{m}=+$ 1 and $\Delta \mathrm{m}=-1$ transitions in the specimen electronic states, where $\mathrm{m}$ is the magnetic quantum number. As orbital angular momentum is conserved in the region of the specimen, any specimen state $\Delta \mathrm{m}$ must produce the opposite $\Delta \mathrm{m}=\mathrm{m}_{\mathrm{f}}-\mathrm{m}_{\mathrm{i}}$ in the electron probe beam. If we prepare a standard STEM probe beam with $\mathrm{m}_{\mathrm{i}}=0$, we can thus measure dichroism in differences in the outgoing electron energy loss spectrum of $\mathrm{m}_{\mathrm{f}}=+1$ and $\mathrm{m}_{\mathrm{f}}=-1$ components of the probe after the specimen by selectively passing more of one of these components at a time into the electron energy loss spectrometer. We propose to use the orbital angular momentum-dependent lensing effect to perform this mode selection.

Mode selection using orbital angular momentum-dependent lensing is quite simple in principle. In a confocal configuration, one can project a slightly defocused image of the probe onto a small aperture (Figures 1 and 2) and pass the apertured probe into an electron energy loss spectrometer. Because of the orbital angular momentum-dependent lensing effect, the aperture will preferentially pass one orbital angular momentum mode over the other. When the lens field is switched, the opposite selection will occur. The difference between energy loss spectra produced this way with opposite lens polarities is a dichroism spectrum.

If successful, this dichroism experiment may enable atomic-resolution structural chirality determination and molecular fingerprinting [8] in addition to out-of-plane magnetization measurement. [9] 
References:

[1] P Schattschneider et al., Nature 441, (2006) p. 486.

[2] J Rusz, JC Idrobo and S Bhowmick, Phys. Rev. Lett 113 (2014), p. 145501.

[3] S Lloyd et al., Phys. Rev. Lett. 108 (2012), p. 074802.

[4] P Schattschneider et al., Phys. Rev. Lett. 110 (2013), p. 189501.

[5] P Schattschneider et al., Ultramicroscopy 136 (2014) p. 81.

[6] J Rusz and S Bhowmick, Phys. Rev. Lett. 111 (2013) p. 105504.

[7] T Harvey et al., Microsc. Microanal. 21 (Suppl 3) (2015), p. 23.

[8] T Harvey at al., arXiv:1507.01810 (2015).

[9] This work was supported by the U.S. Department of Energy (DOE), Office of Science, Basic Energy Sciences (BES), under the Early Career Research Program Award \# DE-SC0010466.

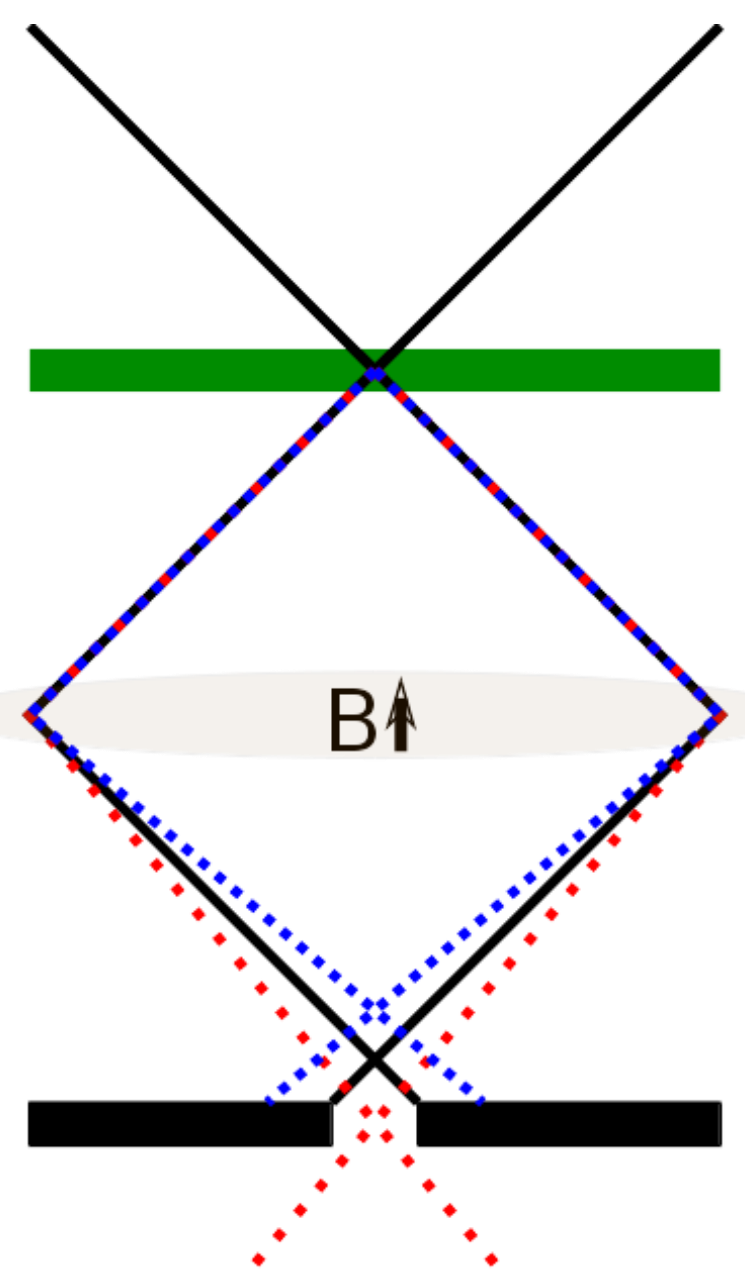

Figure 1. Dichroism setup with lens field pointed up. After the probe interacts with the specimen (green), the +1 (red) OAM component is selectively passed by the aperture (black) relative to the -1 (blue) OAM component.

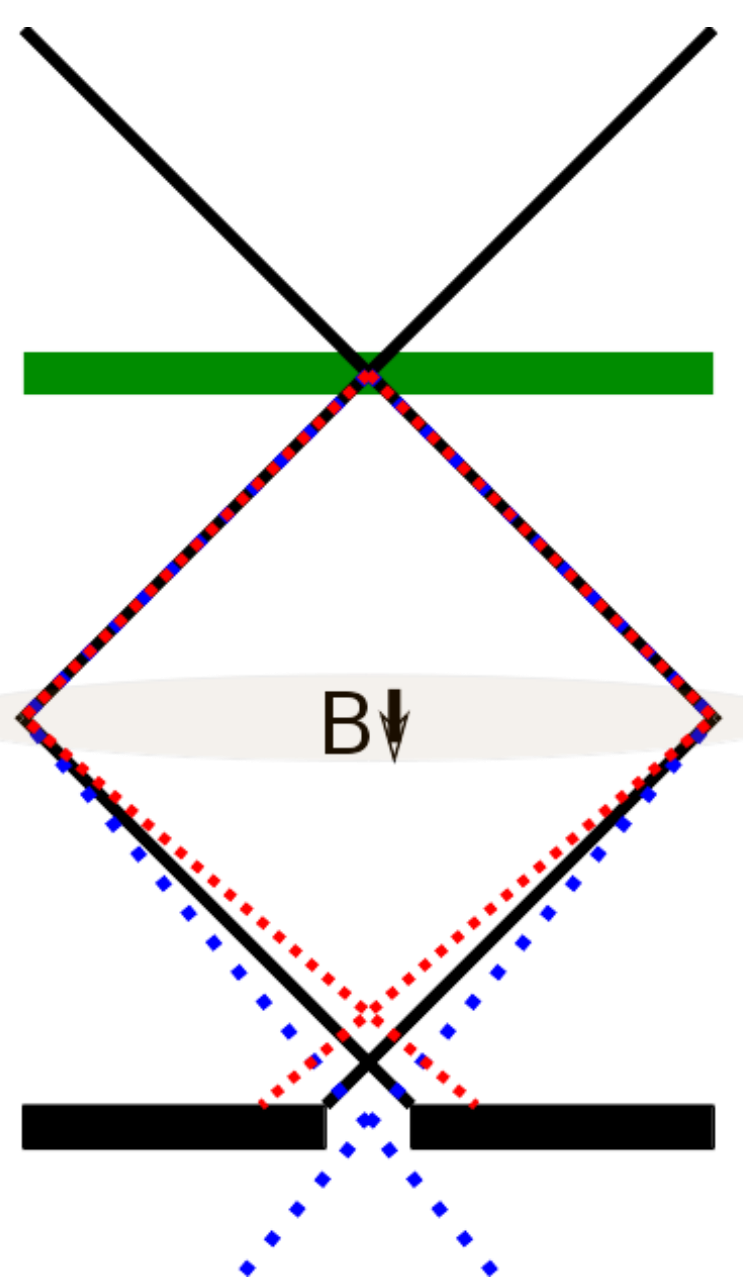

Figure 2. Dichroism setup with lens field pointed down. After the probe interacts with the specimen (green), the -1 (blue) OAM component is selectively passed by the aperture (black) relative to the +1 (red) OAM component. 